\title{
Performance of Zucker rats in two consummatory contrast paradigms
}

\author{
CHARLES F. FLAHERTY and KATHLEEN L. KRAUSS \\ Rutgers University, New Brunswick, New Jersey \\ and \\ WENDY HILL \\ Lafayette College, Easton, Pennsylvania
}

\begin{abstract}
Zucker obese rats showed larger successive negative contrast (degree of reduction in licking when shifted from $32 \%$ to $4 \%$ sucrose) and larger anticipatory contrast (depressed intake of $4 \%$ sucrose when $4 \%$ sucrose preceded $32 \%$ sucrose in once-per-day pairings) than Zucker lean rats. However, the difference between the lines was small and the performance of the obese rats was similar to that of commercially available Sprague-Dawley rats. The lean and obese rats did not differ in their intake of $32 \%$ or $4 \%$ sucrose in noncontrast conditions, but they did differ in openfield activity-the obese rats were considerably less active than the lean rats. These data indicate that Zucker obese rats show normal incentive relativity functions in the consumption of sucrose.
\end{abstract}

Genetically obese Zucker fatty rats differ from Zucker lean rats in a variety of ways, including hyperphagia, hyperinsulinemia, excess adiposity, and hyperlipidemia in the obese rat as compared with the lean (Argiles, 1989; Bray 1977). Zucker obese rats consume a diet higher in fat and lower in protein than that consumed by the lean rats (Castonguay, Hartman, Fitzpatrick, \& Stern, 1982; Castonguay, Rowland, \& Stern, 1985). The obese rats are also more sensitive to the appetite-enhancing effects of glucocorticoids than are the lean rats (Freedman, Castonguay, \& Stern, 1985). The lean and obese rats also differ in binding activity of both $\alpha_{1}$ - and $\alpha_{2}$-noradrenergic receptors in the hypothalamus, but it is not clear whether this is a cause or a consequence of weight differences (Jhanwar-Uniyal, Awad, Gearhart, Finkelstein, \& Leibowitz, 1991).

Although Zucker fatty rats and rats that become obese following ventromedial hypothalamic lesions (VM rats) are both obese, there are behavioral differences between the two preparations. For example, VM rats decrease intake when the diet is diluted with cellulose or adulterated with quinine, but Zucker obese rats adjust their intake to maintain body weight (Bray, 1977). Also, unlike VM rats, Zucker obese rats behave much as do normal rats when operant requirements for food are increased (Greenwood, Quartermain, Johnson, Cruce, \& Hirsch, 1974).

In the present experiments, we compared Zucker lean and obese rats in a different test of food motivation-the negative contrast effect that occurs when the concentra-

The assistance of Cynthia Coppotelli and Jennifer Flaherty in these experiments is appreciated. The research was supported by a grant from the Charles and Johanna Busch Bequest and by the Dean of Arts and Sciences at Rutgers. Address correspondence to C. Flaherty, Psychology Department, Busch Campus, Rutgers University, New Brunswick, NJ 08903. tion of a sucrose solution is decreased. Previous experiments have shown that some selected strains differ in regard to this contrast effect. For example, Syracuse highavoidance rats (SHA) have shown substantially smaller negative contrast effects than Syracuse low-avoidance (SLA) rats (Flaherty \& Rowan, 1989). Also, Maudsley reactive rats (MRA) have shown smaller contrast effects than Maudsley nonreactive rats (MNRA), but both of these strains have shown smaller contrast than unselected rats (Rowan \& Flaherty, 1991).

The available literature on Zucker rats suggests that the obese rats may be similar to unselected rats in contrast (because of the response to adulterated diets), but this literature does not provide a basis for predicting a difference between the lean and obese rats in sucrose contrast experiments. In a previous experiment, the obese rats consumed more of a $32 \%$ sucrose solution than the lean rats, but this finding was under conditions in which the sucrose was a dietary component and not when it was available for brief periods, as in the contrast procedure (Castonguay et al., 1985).

In the present paper we report two experiments in which the behavior of obese and lean Zucker rats was examined in two different contrast paradigms. In Experiment 1 the animals were tested in successive negative contrast-a procedure in which the rats were given brief access to $32 \%$ sucrose for 10 days and then were shifted to $4 \%$ sucrose. The performance of the shifted rats was compared with that of the rats given access to $4 \%$ sucrose only. In Experiment 2 the rats were tested in anticipatory contrast-a procedure in which one group of rats was given brief access to $4 \%$ sucrose followed by brief access to $32 \%$ sucrose in once-per-day pairings. The performance of this group was compared with that of the rats that had received access to the $4 \%$ solution twice each day. 
These two contrast procedures may reflect different incentive relativity mechanisms-successive contrast, reflecting behaviors that occur following abrupt reward decrement, and anticipatory contrast, reflecting behaviors that occur in preparation for the impending occurrence of a substance preferred to the currently available substance. That these processes are different is suggested by the finding that Sprague-Dawley rats, selectively bred to differ in successive negative contrast, do not differ in anticipatory contrast (Flaherty, Krauss, Rowan \& Grigson, 1994). Also, drugs such as chlordiazepoxide and cyproheptadine, which moderate successive negative contrast (Flaherty, Grigson, \& Lind, 1990; Grigson \& Flaherty, 1991), do not affect anticipatory contrast (Flaherty \& Rowan, 1988; Flaherty, Turovsky, \& Krauss, in press).

\section{METHOD}

\section{Experiment 1: Successive Contrast}

The subjects were 23 male, Zucker rats ( 12 obese [ $\mathrm{fa} / \mathrm{fa}$ ] and 11 lean $[\mathrm{Fa} /$ ?]) originally purchased from Charles River Laboratories, Kingston, NY. The rats were initially maintained at Lafayette College, where they were used in a glycemic conditioning experiment. This experiment involved the intraperitoneal administration of insulin (1.0 IU per $316 \mathrm{~g}$ of body weight) paired with a specific stimulus context and the withdrawal of blood from the tail to test for blood glucose levels. No conditioning was obtained in this experiment and there was no difference in results between the obese and lean Zucker rats (W. Hill, unpublished data).

After 2 months at Lafayette College, the rats were moved to Rutgers and maintained on free food and water for 30 days prior to the start of the present experiments. At the start of these experiments the rats were approximately 180 days old. They were reduced to $82 \%$ of their free-feeding weights $(605 \mathrm{~g}$ for the obese and $402 \mathrm{~g}$ for the lean rats) by once-per-day feedings of Purina Rat Chow and a continuous supply of water. The animals were housed singly and under a 14:10-h light:dark cycle. The experiment was conducted at $0900 \mathrm{~h}, 1 \mathrm{~h}$ after light onset in the colony room. Testing was conducted in six Plexiglas chambers with retractable tubes for presenting sucrose solutions (described in Flaherty et al., 1990).

The rats were weighed/handled for 5 days and then exposed to the apparatus for a 5-min habituation period. The rats were then randomly assigned to either a shifted or an unshifted group. The shifted group received access to $32 \%$ sucrose solution for $5 \mathrm{~min}$ a day for 10 days. On Days 11-14 of sucrose access, the shifted rats were given $4 \%$ sucrose for $5 \mathrm{~min}$ each day. The unshifted rats received $4 \%$ sucrose solution on all days. Sucrose solutions were prepared by weight (sucrose/[sucrose + water]), using cane sugar and tap water. The rats were fed $30 \mathrm{~min}$ after each test session.

\section{Experiment 2: Anticipatory Contrast}

The same rats were used again. They were redeprived to $82 \%$ of their free-feeding weights after 45 days on ad-lib food. For this experiment all the rats were approximately 260 days old.

The apparatus, which was similar to that used in Experiment 1, is described in Flaherty, Grigson, Checke, and Hnat (1991).

The experiment was designed as a $2 \times 2 \times 2$ factorial, varying the two Zucker lines (obese or lean), contrast (4\% sucrose followed by $4 \%$ sucrose, or $4 \%$ sucrose followed by $32 \%$ sucrose), and intersolution interval (ISI-5 min or $15 \mathrm{sec}$ ). Previous experience in the successive negative contrast experiment (shifted vs. unshifted conditions) was balanced across groups in assignment to the conditions of Experiment 2.

Two spatially separated tubes were presented in succession once per day for 14 days. Each tube was available for a 3-min period; the time between the availability of each tube (ISI) was $15 \mathrm{sec}$ for one set of animals and $5 \mathrm{~min}$ for the other set. All of the animals received access to the $4 \%$ sucrose in the first tube; half received $4 \%$ in the second tube and half received $32 \%$.

Sixteen days following completion of this experiment, the animals were tested for $4 \mathrm{~min}$ in a copy of the Broadhurst open field (Rowan \& Flaherty, 1991).

\section{RESULTS}

\section{Successive Contrast}

Preshift. There was no reliable difference between the obese and lean rats in terminal lick frequency during the preshift phase when the rats were exposed to either $32 \%$ or $4 \%$ sucrose [strain, $F(1,19)=1.45, p>.05$; strain $\times$ sucrose, $F<1.00$ ]. There was also no overall effect of sucrose concentration $(F<1.00)-$ a result not unique to Zucker rats (Flaherty \& Rowan, 1989; Rowan \& Flaherty, 1991).

Postshift. Postshift performance was analyzed in terms of lick frequency (Figure 1) and proportion of preshift

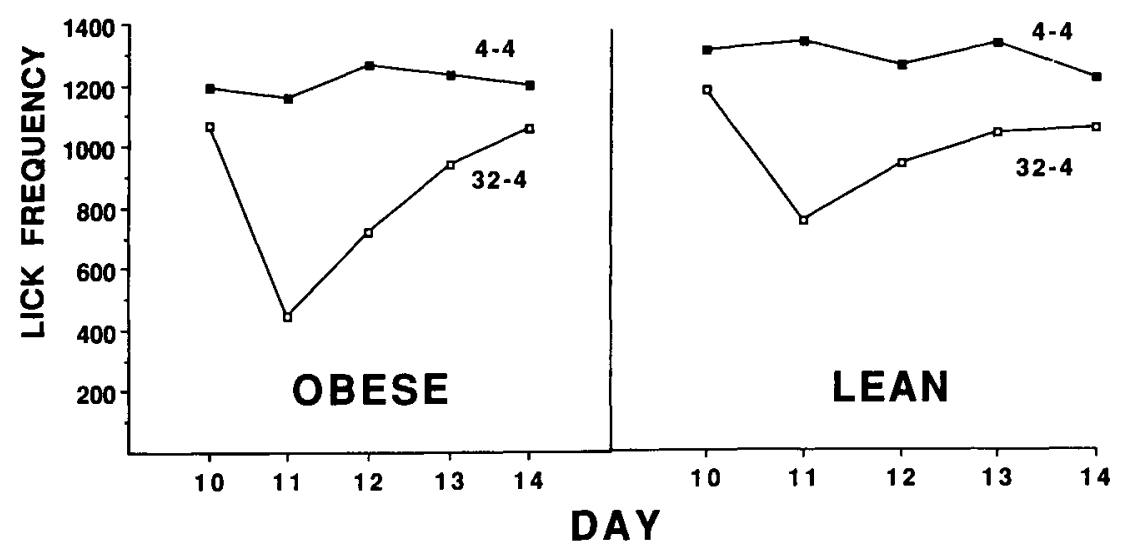

Figure 1. Mean lick frequency of shifted (32-4) and unshifted (4-4) rats on the last preshift day (Day 10) and 4 postshift days. Data from the Zucker obese rats are presented in the left panel and data from the Zucker lean rats are presented in the right panel. 


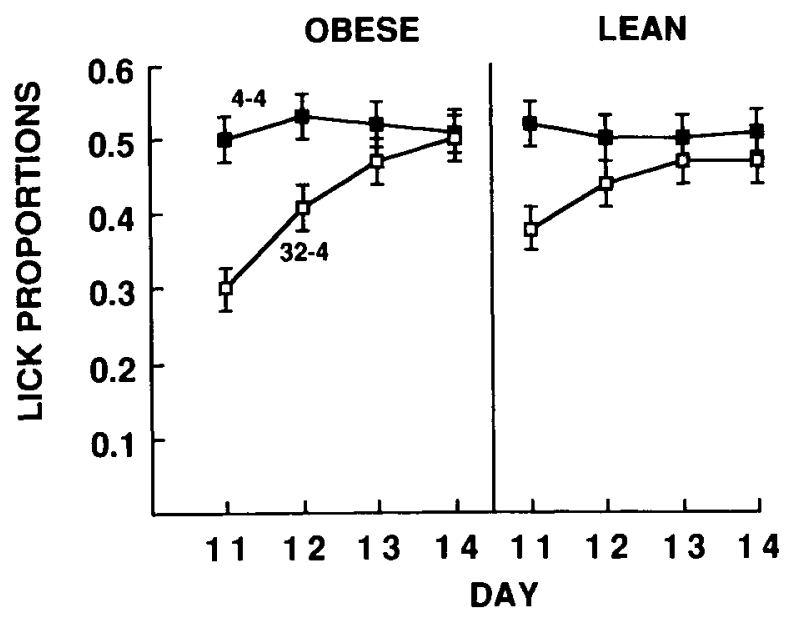

Figure 2. Mean proportions of lick frequency (licks on each postshift day divided by preshift plus appropriate postshift day licks) for both the obese and lean rats under shifted (32-4) and unshifted (4-4) conditions.

lick frequency (Figure 2). The proportion measure adjusts for individual differences in preshift lick frequency.

The shifted rats licked less than the unshifted rats-a negative contrast effect that was reliable in both lick frequency $[F(1,19)=25.35, p<.05]$ and proportion $[F(1,19)=19.25, p<.05]$ measures. There was no overall strain effect in postshift performance [lick frequency, $F(1,19)=1.47, p>.05$; proportion, $F<$ $1.00]$. However, there was a difference between the obese and lean rats across the 4 postshift days; the obese rats showed a larger contrast effect than the lean rats. The effect, though, was subtle and statistical support for this conclusion varied somewhat with the lick frequency and proportion measures. First, there was a reliable strain $X$ day effect with both measures [lick frequency, $F(3,56)=$ 4.67, $p<.05$; proportions, $F(3,55)=5.11, p<.05$ ]. Subsequent analysis with the least significant difference $(L S D)$ procedure $(p<.05)$ indicated that the lean rats licked more than the obese rats on the first 2 postshift days in the lick frequency measure and on the first postshift day in the proportion measure. This difference largely reflected the greater reduction in lick frequency following the shift in the obese rats (see Figure 1). Statistical support for this differential contrast effect was mixed. The sucrose $\times$ strain $\times$ day effect was not reliable in the lick frequency measure $[F(3,56)=2.56, p=.06]$, but it was reliable in the proportion measure $[F(3,55)=4.13, p=$ .01]. Subsequent analyses of the proportion data with the $L S D$ procedure $(p<.05)$ indicated that the obese rats showed a reliable contrast effect on the first 2 postshift days, whereas the lean rats showed a reliable contrast effect on only the first postshift day. Furthermore, the shifted obese rats licked proportionately less than the shifted lean rats on the first postshift day, but not on any other day. The unshifted lean and obese rats did not differ ( $L S D$ test).
In order to place the contrast obtained with the Zucker rats in perspective, the degree of decrement on the first postshift day was compared with similar data obtained with other selected strains tested in the Rutgers laboratory under similar conditions. These data, which are illustrated in Figure 3, indicate that the Zucker obese (FAT) rats showed a larger decrement in lick frequency than both of the Maudsley strains (MNR, MR) and the Syracuse high-avoidance (SHA) strain. But the obese rats showed a smaller decrement than the large-contrast (LC) line, the Syracuse low-avoidance (SLA) strain, and the unselected rats (POP). The Zucker obese rats and the small-contrast (SC) line did not differ.

The Zucker lean rats showed a greater decrement than the Maudsley reactive strain, did not differ from the Maudsley nonreactive strain or the SHA rats, and showed a smaller decrement than all of the other strains. It should be emphasized that the between-strain comparisons are across different experiments at different times, and thus should be interpreted with caution.

\section{Anticipatory Contrast}

With a 15-sec ISI, the obese rats given the 4-32 condition suppressed intake of the $4 \%$ solution in comparison with the 4-4 control group. This anticipatory contrast effect was reliable over Blocks 3-7. The lean rats showed no suppression (no contrast) at all [sucrose $\times$ strain $X$ ISI, $F(6,47)=3.29, p<.01 ; L S D$ tests, $p<.05$ ]. At the 5-min ISI there was an overall reliable contrast effect $[F(1,7)=5.75, p<.05]$ that did not differ for the lean and obese rats $(F<1.00)$ nor across blocks $[F(6,40)=$ $1.07, p>.35]$. These data are illustrated in Figure 4 . There were no differences as a function of sucrose con-

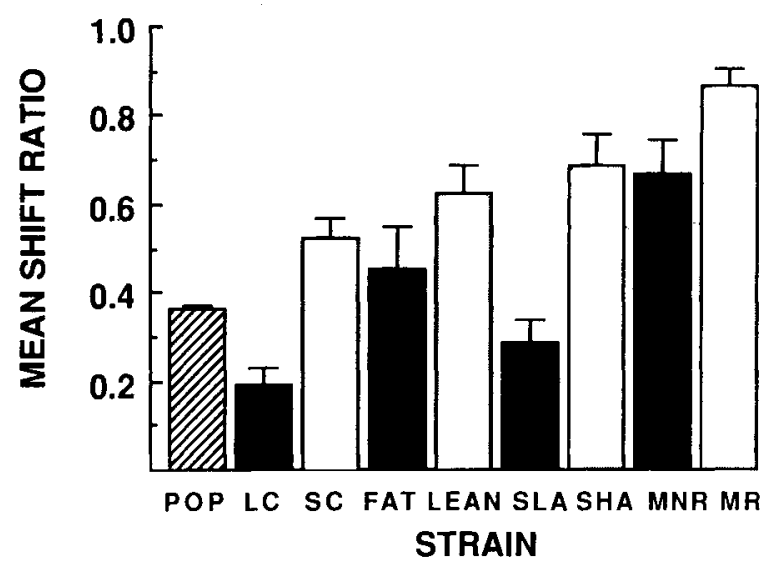

Figure 3. Mean shift ratios (postshift/preshift lick frequency) for each of the selected strains: large contrast (LC), small contrast (SC), Zucker obese (FAT), Zucker lean, Syracuse low-avoidance (SLA), Syracuse high-avoidance (SHA), Maudsley nonreactive (MNR), Maudsley reactive (MR), and a population of 397 unselected rats (POP) tested in our laboratory in the negative contrast paradigm (see text). 

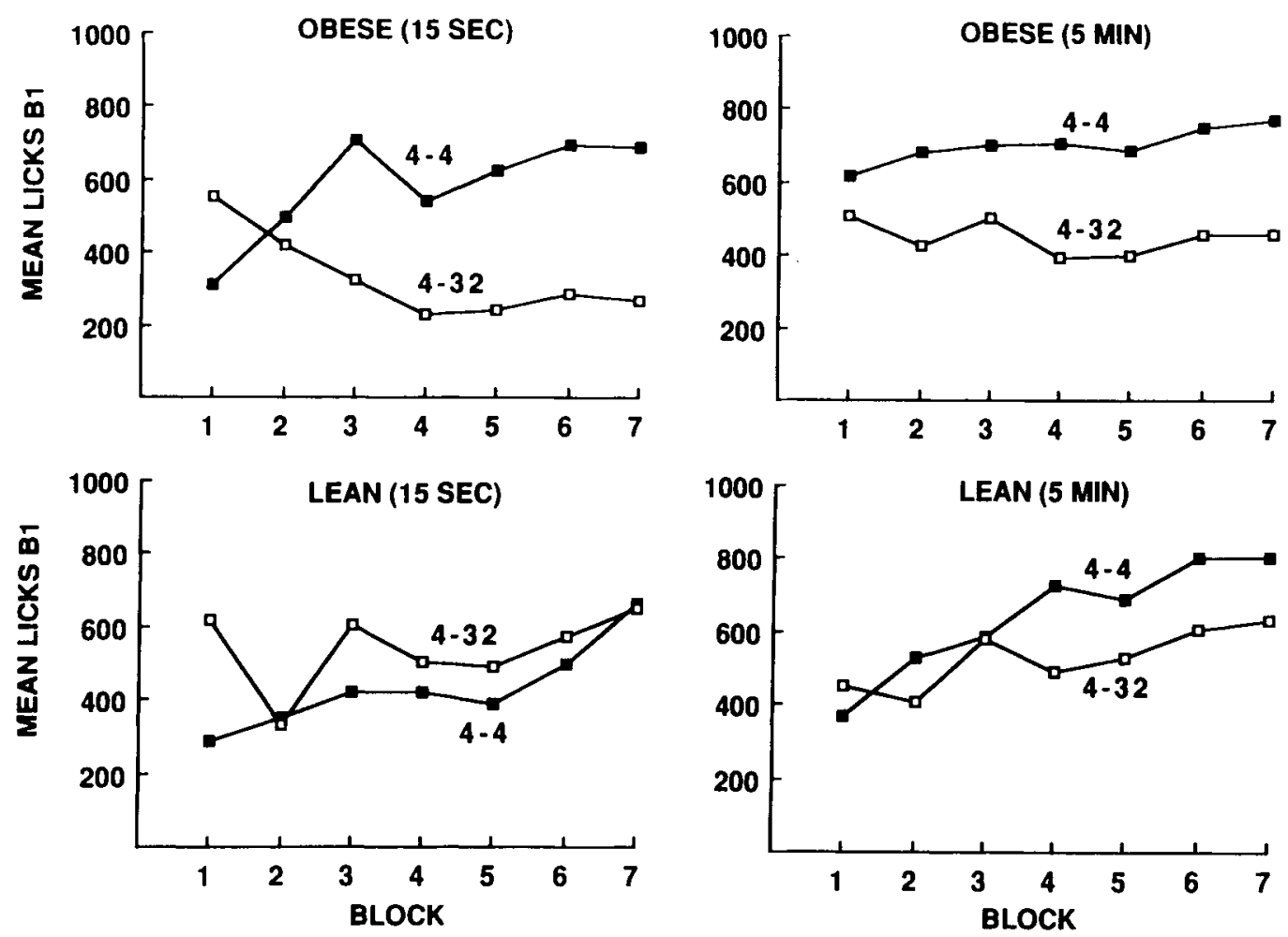

Figure 4. Mean licks on Bottle 1 (4\% sucrose for all groups) for obese and lean rats as a function of both ISI (either 15 sec or $5 \mathrm{~min}$ ) and contrast group (either $4 \%$ or $32 \%$ sucrose in Bottle 2 ).

dition or group in latency to initiate licking for the first solution.

Lick frequency for the second solution available each day revealed that both of the Zucker groups licked more of the $32 \%$ solution than of the $4 \%$ solution $[F(1,8)=$ $6.70, p<.05]$ when there was a 15-sec ISI, but with a 5-min ISI there was no concentration effect $(F<1.00)$.

Analysis of the $\log _{(10)}$ latency data indicated that the rats that received $32 \%$ sucrose initiated licking sooner

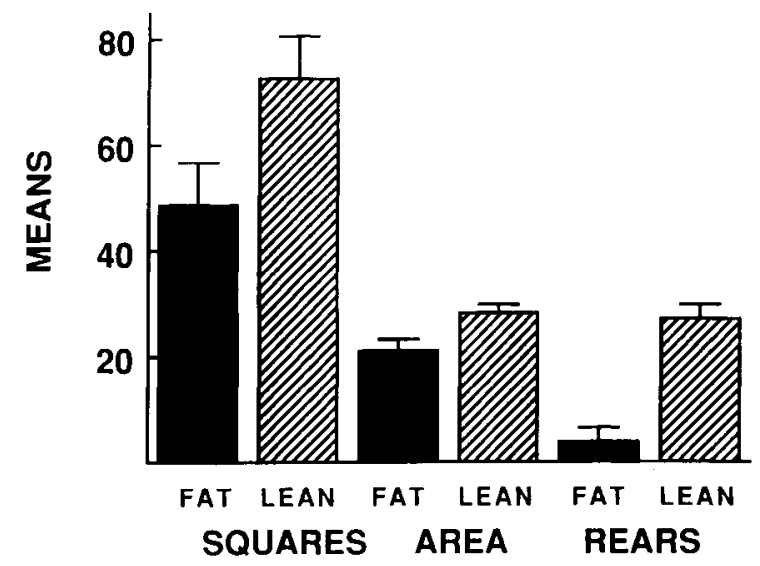

Figure 5. Mean total number of squares entered, number of different squares entered (area), and number of rears for the obese and lean rats in an open field.
$(M=1.73 \mathrm{sec})$ than the rats that received $4 \%$ sucrose $[M=5.75 \mathrm{sec} ; F(1,15)=21.65, p<.05]$. There was no difference between the lean $(M=3.30 \mathrm{sec})$ and obese $(M=2.88 \mathrm{sec})$ rats in latency to initiate licking for the second solution. There was an ISI effect; the group that received $32 \%$ sucrose initiated licking sooner $(M=$ $1.25 \mathrm{sec})$ than the group that received $4 \%(M=8.07 \mathrm{sec})$ when there was a 15-sec ISI, but there was no concentration effect when there was a 5-min ISI [ $32 \%$ group, $M=$ $2.42 \mathrm{sec} ; 4 \%$ group, $M=3.85 \mathrm{sec}$; concentration $\times$ ISI, $F(1,19)=7.13, p<.01 ; L S D$ tests, $p=.05$ ].

\section{Open-Field Data}

As illustrated in Figure 5, the lean rats entered more squares $[F(1,20)=8.60, p<.05]$, were more likely to enter different areas of the field $[F(1,20)=12.13$, $p<.05]$, and reared more often than the obese rats $[F(1,20)=58.95, p<.05]$. There were no differences between the obese and lean rats in defecation, urination, or thigmotaxis.

\section{DISCUSSION}

Although the Zucker lean and obese rats differed in contrast, the lean rats deviated more from the degree of contrast typically obtained in commercially bred, unselected rats. The Zucker lean rats tended to show a smaller successive negative contrast effect than did the obese rats, 
whose performance was similar to that of unselected rats (Figure 3). Anticipatory contrast with $4 \%$ and $32 \%$ sucrose conditions is normally obtained with both 15 -sec and 5-min ISIs when the animals are freely fed (Flaherty et al., 1991), and at $15 \mathrm{sec}$, but not $5 \mathrm{~min}$, in deprived rats (Flaherty et al., 1991). Thus, the lean animals differed from the unselected rats at both ISIs (no contrast at $15 \mathrm{sec}$, contrast at $5 \mathrm{~min}$ ), whereas the obese rats differed in showing a contrast where it normally does not occur-at a 5-min ISI.

To some extent, the behavior of the obese rats (compared with the lean) is consistent with the behavior of rats that are less food deprived, in that successive negative contrast is of longer duration (Dachowski \& Brazier, 1991; Riley \& Dunlap, 1979) and anticipatory contrast occurs at both a 15-sec and a 5-min ISI (Flaherty et al., 1991). Little that is systematic in regard to the previous literature is apparent in the behavior of the lean rats. Their behavior in the successive negative contrast experiment and with a 15-sec ISI in the anticipatory contrast experiment suggests that they are simply less prone to relativity effects. However, with a 5-min ISI, their behavior in the anticipatory contrast experiment was not different from the obese rats, and both lines indicate a greater tendency to contrast at this value than unselected rats (Flaherty et al., 1991).

These results suggest that any dietary/regulatory abnormalities existing in Zucker obese rats do not prevent the occurrence of relativity effects in the consumption of sucrose solutions. Perhaps the use of fats as reinforcers would reveal abnormalities in contrast not detected by carbohydrate manipulations, particularly because Zucker obese rats typically self-select diets higher in fat than Zucker lean rats (Castonguay et al., 1982). Such specificity in reinforcement relativity would be of some interest. It should be noted that the difference in intake of a $32 \%$ sucrose solution reported by Castonguay et al. (1985) was not replicated in the preshift phase of Experiment 1 nor in the Bottle 2 intake data of the anticipatory contrast experiment. The difference between the studies is probably accounted for by the fact that the sucrose was constantly available as a dietary component in Castonguay et al.'s (1985) study, but was only briefly available each day in the present experiments. The failure to find a difference between the Zucker lean and obese rats with brief exposure of the sucrose solutions suggests that the results obtained by Castonguay et al. (1985) might reflect a difference between lean and obese rats in satiation to $32 \%$ sucrose rather than a difference in avidity. The lack of a difference in avidity for sucrose between the lean and obese rats is also supported by the failure to obtain a difference between the two groups in the latency to initiate drinking in Experiment 2, even though there was an effect of sucrose concentration in this measure-the rats that received $32 \%$ sucrose initiated licking sooner than those that received $4 \%$ sucrose.
The finding that the lean rats showed unusually small contrast effects, both in successive and anticipatory contrast (at a 15-sec ISI), is not predicted by any of the available literature on Zucker rats. This suggests that further study of reward and reinforcement functions in the lean and obese Zucker rats is warranted.

The open-field data are interesting in two regards. First, previous observations have suggested that the lean and obese rats do not differ in spontaneous activity (Keesey, Swiergiel, \& Corbett, 1990); these earlier data were obtained from home cage observations of the animals. However, the Zucker groups clearly differed when placed in a brightly illuminated open field. Second, rats specifically selected for successive negative contrast show differences in activity-large-contrast rats enter more squares than small-contrast rats (Flaherty et al., 1994). The present data, showing that Zucker obese rats had a larger contrast effect than did lean rats but were less active in the open field, suggest that there is no necessary relationship between activity level and successive negative contrast.

\section{REFERENCES}

Argiles, J. M. (1989). The obese Zucker rat: A choice for fat metabolism 1968-1988: Twenty years of research on the insights of the Zucker mutation. Progress in Lipid Research, 28, 53-66.

Bray, G. A. (1977). The Zucker-fatty rat: A Review. Federation Proceedings, 36, 148-153.

Castonguay, T. W., Hartman, W. J., Fitzpatrick, E. A., \& Stern, J. S. (1982). Dietary self-selection and the Zucker rat. Joumal of Nutrition, 112, 796-800.

Castonguay, T. W., Rowland, N. E., \& Stern, J. S. (1985). Nutritional influences on dietary selection patterns of obese and lean Zucker rats. Brain Research Bulletin, 14, 625-631.

DAChowski, L., \& Brazier, M. B. (1991). Consummatory incentive contrast: Experimental design relationships and deprivation effects. In L. Dachowski and C. F. Flaherty (Eds.), Current topics in animal leaming: Brain, emotion, and cognition (pp. 245-270). Hillsdale, NJ: Erlbaum.

Flaherty, C. F., Grigson, P. S., Checke, S., \& Hnat, K. C. (1991), Deprivation state and temporal horizons in anticipatory contrast. Journal of Experimental Psychology: Animal Behavior Processes, 17, 503-518.

Flaherty, C. F., Grigson, P. S., \& Lind, S. (1990). Chlordiazepoxide and the moderation of the initial response to reward reduction. Quarterly Journal of Experimental Psychology, 42B, 87-105.

Flaherty, C. F., Krauss, K. L., Rowan, G. A., \& Grigson, P. S. (1994). Selective breeding for negative contrast in consummatory behavior. Journal of Experimental Psychology: Animal Behavior Processes, 20, 3-19.

FlaherTy, C. F., \& Rowan, G. A. (1988). Effect of intersolution interval, chlordiazepoxide, and amphetamine on anticipatory contrast. $A n$ imal Learning \& Behavior, 16, 47-52.

FlaherTy, C. F., \& Rowan, G. A. (1989). SHA and SLA rats differ in novelty stress, glycemic conditioning and incentive contrast. $B e$ havioral \& Neural Biology, 51, 145-164.

Flaherty, C. F., Turovsky, J., \& Krauss, K. L. (in press). Relative hedonic value modulates anticipatory contrast. Physiology \& Behavior.

Freedman, M. R., Castonguay, T. W., \& Stern, J. S. (1985). Effect of adrenalectomy and corticosterone replacement on meal patterns of Zucker rats. American Journal of Physiology, 249, R584-R594.

Greenwood, M. R. C., Quartermain, D., Johnson, P. R., Cruce, 
J. A. F., \& Hirsch, J. (1974). Food motivated behavior in genetically obese and hypothalamic-hyperphagic rats and mice. Physiology \& Behavior, 13, 687-692.

Grigson, P. S., \& Flaherty, C. F. (1991). Cyproheptadine prevents the initial occurrence of successive negative contrast. Pharmacology, Biochemistry \& Behavior, 40, 433-442.

Jhanwar-Uniyal, M., Awad, I. R., Gearhart, G. M., Finkelstein, J. A., \& Leibowitz, S. F. (1991). Higher alpha-noradrenergic receptors in paraventricular nucleus of obese Zucker rats: Decline after food deprivation. Pharmacology, Biochemistry \& Behavior, 40, 853-859.

Keesey, R. E., Swiergiel, A. H., \& Corbett, S. W. (1990). Contri- bution of spontaneous activity to daily energy expenditure of adult obese and lean Zucker rats. Physiology \& Behavior, 48, 327-331. Riley, E. P., \& DunlaP, W. P. (1979). Successive negative contrast effects as a function of deprivation condition following shifts in sucrose concentration. American Journal of Psychology, 92, 59-70.

Rowan, G. A., FlaherTy, C. F. (1991). Behavior of Maudsley reactive and nonreactive rats (Rattus norvegicus) in three consummatory contrast paradigms. Journal of Comparative Psychology, 105, 115-124.

(Manuscript received October 13, 1992; revision accepted for publication November 22, 1993.) 